\title{
Kernos
}

Revue internationale et pluridisciplinaire de religion grecque antique

18 | 2005

Varia

\section{Laurent BRICAULT, Isis en Occident. Actes du II ème colloque international sur les études isiaques, Lyon III, 16-17 mai 2002}

\section{Richard Veymiers}

\section{(2) OpenEdition \\ Journals}

Édition électronique

URL : http://journals.openedition.org/kernos/1732

DOI : 10.4000/kernos. 1732

ISSN : 2034-7871

\section{Éditeur}

Centre international d'étude de la religion grecque antique

\section{Édition imprimée}

Date de publication : 1 janvier 2005

Pagination : $549-550$

ISSN : 0776-3824

\section{Référence électronique}

Richard Veymiers, «Laurent BRICAuLt, Isis en Occident. Actes du Ilème colloque international sur les études isiaques, Lyon III, 16-17 mai $2002 »$, Kernos [En ligne], 18| 2005, mis en ligne le 24 mai 2011, consulté le 21 septembre 2020. URL : http://journals.openedition.org/kernos/1732 ; DOI : https:// doi.org/10.4000/kernos. 1732 
La troisième et dernière section de l'ouvrage s'intéresse plus spécifiquement à la confrontation de la religion gréco-romaine avec les cultures locales et aux processus d'acculturation. On ne s'étonnera pas que la moitié des communications sur ce thème concernent l'Égypte qui offre, comme l'on sait, une matière privilégiée pour des études concernant les phénomènes interculturels : J.-C. Goyon met en valeur le rôle joué par l'élite sacerdotale dans le sud de l'Égypte, en liaison avec les cultes d'Isis et d'Horus à Philae et Edfou; M. Drew-Bear présente de façon convaincante, en prenant l'exemple de la Moyenne Égypte, les liens qu'on peut établir entre toponymes et cultes locaux traditionnels; P. Schubert exploite un dossier documentaire de la collection papyrologique de Genève pour éclairer la façon dont les autorités civiles romaines et le clergé égyptien étaient amenés à collaborer dans des procédures relatives à des cultes locaux. Deux contributions traitent de l'influence anatolienne décelable dans des cultes d'Asie Mineure, au Métrôon d'éphèse (C.M. Thomas) et en Lycie (Chr. Le Roy). N. Jijina évoque pour sa part, en anglais, le mélange des cultures que révèle dans la région du Bosphore une étude de l'art funéraire.

L'ensemble constitue un recueil dont la lecture est stimulante et où, grâce à un index détaillé, il sera possible d'effectuer des parcours croisés à partir des sources, tant littéraires qu'épigraphiques, des figures héroïques ou divines et des noms, historiques, mythiques ou géographiques. Mais il me semble que le caractère quelque peu hétéroclite de l'ouvrage aurait gagné à être corrigé par des remarques introductives plus développées, visant à mieux circonscrire les enjeux d'une réflexion portant sur une très vaste thématique.

Yves Lafond

(Université de Poitiers)

Laurent Bricault (éd.), Isis en Occident. Actes du II ${ }^{\text {ime }}$ colloque international sur les études isiaques, Lyon III, 16-17 mai 2002, Leiden, Brill, 2004. 1 vol. $16 \times 24 \mathrm{~cm}, \mathrm{XXV}+510$ p. (Religions in the Graeco-Roman World, 151). ISBN : 90-04-13263-5.

Les actes de ce second colloque international, riches de dix-huit contributions, rédigées sur plus de cinq cents pages en français, anglais, allemand et italien par des chercheurs issus d'horizons très variés, participent du renouveau noté dans la recherche isiaque depuis ces dernières années. Bien que traitant de recherches particulières, les articles se rattachent à certains axes principaux retenus pour peindre un tableau actualisé de la diffusion d'Isis en Occident.

Une première série de communications propose des études régionales permettant de faire le point sur les conditions d'implantation et de diffusion des cultes isiaques dans plusieurs zones de l'Occident méditerranéen. Grâce à ces enquêtes géographiques, réalisées dans le cadre de l'Italie (M. Malaise), de l'Hispanie (J. Alvar, E. Muñiz), de la Gaule (J. Leclant), des deux Germanies (M. Haase), des provinces alpines (S. Cibu, B. Rémy), du limes danubien (M.-Ch. Budischovsky) et de l'Afrique du Nord (L. Bricault, Y. Le Bohec, J.-L. Podvin sur la Proconsulaire; J.-P. Laporte sur la Maurétanie Césarienne, Numidie et partie de la Proconsulaire; Y. Le Bohec sur la Maurétanie Tingitane), il est possible d'aborder les grandes questions de la diffusion de ces cultes et de procéder à d'intéressantes comparaisons. Ainsi, nous pouvons observer la nature des itinéraires empruntés par les divinités isiaques, l'époque de leur implantation, l'identité des agents de leur diffusion, issus par exemple du monde militaire (A. Perrissin-Fabert), ainsi que leur succès dans les populations autochtones. Selon le territoire envisagé, la répartition des isiaca est inégale et les membres du cercle isiaque 
ont une importance relative entre eux. Les témoignages de dévotion isiaque sont parfois difficiles à identifier tant la frontière entre le culturel et le cultuel peut être floue. Cette ambiguité révèle la nécessité d’un consensus dans la terminologie utilisée par les spécialistes. Qu'entend-on par exemple par site isiaque, par isiaca ou par Aegyptiaca?

Deux travaux se concentrent sur des sites particuliers considérés comme des centres isiaques majeurs et tentent de préciser la chronologie et le statut de certains sanctuaires. L'un (S. Ensoli) présente les fouilles exécutées à Cyrène dans le temple acropolitain d'Isis et de Sérapis. L'autre (M.J. Versluys) concerne l'existence possible d'un Iséum sur le Capitole romain dès le Ir siècle av. J.-C.

Plusieurs enquêtes portent sur certaines catégories documentaires dont l'étude avait souvent jusqu'ici été sous-estimée et négligée : les lampes fabriquées hors d'Égypte (J.L. Podvin), les monnaies grecques d'Ionie et de Carie (F. Delrieux), les intailles et gemmes magiques (C. Sfameni), ainsi que les formes décoratives des sanctuaires (K. Parlasca). Tous ces documents présentent un potentiel informatif considérable qu'il convient de réhabiliter pour autoriser une vision cohérente et complète du phénomène isiaque. Ils possèdent évidemment un degré de signification variable et ne sont pas dénués de problèmes d'interprétation.

Enfin, le volume s'achève par une conclusion (M. Malaise) qui reprend les grands thèmes du colloque, mais qui soulève aussi d'autres questions et énonce de nouveaux souhaits visant à améliorer notre connaissance d'Isis en Occident.

Richard Veymiers

(FNRS - Université de Liège)

Monique Bouquet, Françoise Morzadec (éds), La Sibylle. Parole et représentation, Rennes, Presses Universitaires de Rennes, 2004. 1 vol. $15 \times 21 \mathrm{~cm}, 301$ p. (Collection «Interférences »). ISBN : 2-86847-879-4.

La voix de la Sibylle conserve toujours sa sonorité. Ce volume, qui réunit le contributions au colloque tenu à l'Université de Rennes 2-Haute Bretagne en octobre 2001 (organisé par le Centre d'Études de Littératures Antiques et Modernes), en est un bon exemple. Étant donné que les dernières années ont connu une sorte de fièvre sibylline dans le monde éditorial, le premier éloge qui mérite l'ouvrage est qu'il a sa propre personnalité et que, même lorsqu'on y traite des aspects plus connus, les A. ont trouvé des points de vue originaux et des perspectives nouvelles. De surcroit, une bonne partie des contributions met en évidence des aspects très peu connus de l'univers sibyllin. C'est donc un ensemble qui a une place très méritoire dans la production bibliographique sibylline. Voici un résumé (nécessairement trop télégraphique) du contenu.

L'Avant-propos de M. Bouquet et F. Morzadec, p. 11-16 (avec une description, brève mais précise, de l'ensemble) ouvre le chemin aux chapitres suivants. Caroline Février, «Le double langage de la Sibylle : de l'oracle grec au rituel romain », p. 17-28 : intéressante hypothèse sur l'usage que les décemvirs faisaient des libri sibyllini pour la procuration des prodiges comme moyen de rénovation des pratiques rituelles. Charles Guittard, "Reflets étrusques sur la Sibylle : Libri Sibyllini et Libri Vegoici», p. 29-42: sur les rapprochements entre les livres sibyllins et l'etrusca disciplina et le rôle de la figure de la lasa Vecu (Végoia). Jacqueline Champeaux, "Figures romaines de la Sibylle», p. 43- 52 : Virgile a fusionné tous les types sibyllins précédents et, comme résultat, a créé une figure «innovante, totale et unitaire» (p. 46) qui aura une énorme influence. Christophe Cusset, «Cassandra et/ou la sibylle : les voix dans l'Alexandra 